\title{
A Model of Dark Matter and Dark Energy Based on Relativizing Newton's Physics
}

\author{
Ramzi Suleiman1,2,3,4 \\ ${ }^{1}$ Triangle Research \& Development Center (TRDC), KfarQara, Israel \\ ${ }^{2}$ Acuura-c LTD, Hiafa, Israel \\ ${ }^{3}$ Department of Psychology, University of Haifa, Israel, Israel \\ ${ }^{4}$ Department of Philosophy, Al Quds University, Al-Bireh, Palestine \\ Email: suleiman@psy.haifa.ac.il
}

How to cite this paper: Suleiman, R. (2018) A Model of Dark Matter and Dark Energy Based on Relativizing Newton's Physics. World Journal of Condensed Matter Physics, 8, 130-155.

https://doi.org/10.4236/wjcmp.2018.83009

Received: May 19, 2018

Accepted: August 28, 2018

Published: August 31, 2018

Copyright $\odot 2018$ by author and Scientific Research Publishing Inc. This work is licensed under the Creative Commons Attribution International License (CC BY 4.0).

http://creativecommons.org/licenses/by/4.0/

(c) (i) Open Access

\begin{abstract}
The nature and properties of dark matter and dark energy in the universe are among the outstanding open issues of modern cosmology. Despite extensive theoretical and empirical efforts, the question "what is dark matter made of?" has not been answered satisfactorily. Candidates proposed to identify particle dark matter span over ninety orders of magnitude in mass, from ultra-light bosons, to massive black holes. Dark energy is a greater enigma. It is believed to be some kind of negative vacuum energy, responsible for driving galaxies apart in accelerated motion. In this article we take a relativistic approach in theorizing about dark matter and dark energy. Our approach is based on our recently proposed Information Relativity theory. Rather than theorizing about the identities of particle dark matter candidates, we investigate the relativistic effects on large scale celestial structures at their recession from an observer on Earth. We analyze a simplified model of the universe, in which large scale celestial bodies, like galaxies and galaxy clusters, are non-charged compact bodies that recede rectilinearly along the line-of-sight of an observer on Earth. We neglect contributions to dark matter caused by the rotation of celestial structures (e.g., the rotation of galaxies) and of their constituents (e.g., rotations of stars inside galaxies). We define the mass of dark matter as the complimentary portion of the derived relativistic mass, such that at any given recession velocity the sum of the two is equal to the Newtonian mass. The emerging picture from our analysis could be summarized as follows: 1) At any given redshift, the dark matter of a receding body exists in duality to its observable matter. 2) The dynamical interaction between the dark and the observed matter is determined by the body's recession velocity (or redshift). 3) The observable matter mass density decreases with its recession velocity, with matter transforming to dark matter. 4) For redshifts $z<0.5$, the universe
\end{abstract}


is dominated by matter, while for redshifts $z>0.5$ the universe is dominated by dark matter. 5) Consistent with observational data, at redshift $z=0.5$, the densities of matter and dark matter in the universe are predicted to be equal. 6) At redshift equaling the Golden Ratio $(z \approx 1.618)$, baryonic matter undergoes a quantum phase transition. The universe at higher redshifts is comprised of a dominant dark matter alongside with quantum matter. 7) Contrary to the current conjecture that dark energy is a negative vacuum energy that might interact with dark matter, comparisons of our theoretical results with observational results of $\Lambda \mathrm{CDM}$ cosmologies, and with observations of the relative densities of matter and dark energy at redshift $z \approx 0.55$, allow us to conclude that dark energy is the energy carried by dark matter. 8) Application of the model to the case of rotating bodies, which will be discussed in detail in a subsequent paper, raises the intriguing possibility that the gravitational force between two bodies of mass is mediated by the entanglement of their dark matter components.

\section{Keywords}

Dark Matter, Dark Energy, GZK Cosmic Rays Cutoff, $\Lambda$ CDM Cosmology, Coincidence Problem, Quantum Phase Transition, Gravity, Golden Ratio

\section{Introduction}

The nature of Dark Matter is one of the most pressing open problems of modern cosmology [1] [2] [3]. Today, evidence for its existence is firmly established due to a wide array of independent experiments and observations [1] [2] [4] [5] [6]. The proposed candidates to explain the nature of dark matter span over 90 orders of magnitude in mass, from ultra-light bosons to massive black holes [1] [7] [8] [9]. A popular class of dark matter candidates are weakly interacting massive particles (WIMPs) [10] [11] [12] [13]. Neutrinos are weakly interacting particles that were almost certainly created in great abundance during the big bang. Although they are believed to constitute part of the dark matter, they are one of numerous other candidates that were and are still being considered. Some candidates for dark matter, like neutrinos and axions, are included in the Standard Model, but most WIMPs candidates come from beyond it. The most widely studied candidate beyond the Standard Model is probably the supersymmetric neutralino. Other supersymmetric candidates for dark matter include zxinos (the supersymmetric partner of the axion), and gravitinos.

Another popular class of dark matter candidates are baryonic matter tied up in brown dwarfs or in chunks of massive compact halo objects, or MACHOs [14] [15] [16], including primordial black holes (PBHs) [17].

Dark energy remains a complete mystery. The common prejudice is that it is some unknown substance, with an enormous anti-gravitational force, which drives the galaxies of our universe apart in accelerated motion [10] [18] [19]. 
One explanation for dark energy is founded on Einstein's Cosmological Constant $(\Lambda)$. According to this explanation the universe is permeated by a negative energy density, constant in time and uniform in space. The big problem with this explanation is that for $\Lambda \neq 1$, it requires that the magnitude of $\Lambda$ be $\approx 10^{120}$ times the measured ratio of pressure to energy density. An alternative explanation argues that dark energy is an unknown dynamical fluid, i.e., one with a state equation that is dynamic in time, $\Lambda=\Lambda(\mathrm{t})$. This type of explanation is represented by theories and models that differ in their assumptions regarding the nature of the state equation dynamics [20] [21] [22] [23]. This explanation is no less problematic than the first one, as it entails the prediction of new particles with masses thirty-five orders of magnitude smaller than the electron mass, which might imply the existence of yet unknown forces. At present there is no persuasive theoretical explanation for the existence and dynamics of dark energy. Although recent research indicates that dark matter and dark energy interact with each other [24] [25], most physicists believe that dark energy is a completely different entity than dark matter, and that the two are uncorrelated.

Given the lack of knowledge about the nature of dark matter and dark energy, most experts contend that understanding the content of the universe and its cosmic acceleration requires nothing less than discovering a new physics. As an example, the Dark Energy Task Force (DETF) summarized its 2006 comprehensive report on dark energy by stating that there is consensus among most physicists that "nothing short of a revolution in our understanding of fundamental physics will be required to achieve a full understanding of the cosmic acceleration" [18] (p. 6). The question of dark matter is important not only for cosmology and astrophysics; it is of great importance to particle physics. Since the Standard Model does not provide a satisfactory dark matter candidate, many contend that dark matter is therefore evidence for physics beyond the Standard Model [1].

Here we take a completely different approach than all contemporary physics to explain dark matter, dark energy, and the interaction between matter and dark matter in the universe. Our approach is relativistic, based on our recently proposed Information Relativity theory (previously termed Complete Relativity theory) [26] [27] [28]. The theory, described briefly in Section 2, is an axiom-free "relativizing" of Newtonian physics, accomplished only by account for the time travel of waves (e.g., light) from one reference frame to another. An important feature of the theory is that it applies to all bodies of mass, regardless of their size, mass, and composition. Hence we will not entertain questions pertaining to the Standard Model of elementary particles, nor to its many extensions. As will be clear in the proceedings, the question that we shall attempt to answer is not what particles may constitute dark matter, but rather how dark matter and matter coexist and interact, and what parameters affect their relative energy densities in the universe. With regard to dark energy, the surprising possibility arising from our analysis is that it is nothing but the energy carried 
by dark matter.

The following sections are organized as follows: In section 2 we summarize the main tenets of Information Relativity theory, and depict its main transformations. In section 3 we apply the theory to a simplified model of the Universe, in which large-scale celestial bodies, like galaxies and galaxy clusters, are compact bodies receding rectilinearly along the line-of-sight of an observer on Earth. In our simplistic model we neglect contributions to dark matter caused by the rotation of celestial structures (e.g., the rotation of galaxies), and of their constituents (e.g., rotation of stars inside galaxies). For such a grossly simplified universe, we define dark matter, and derive exact terms for the dynamics between matter and dark matter densities, and of their respective energy distributions in the universe, as functions of the recession velocity. In section 4 we express the derived terms as functions of the redshift $z$, and utilize the theoretical results to explain the GZK cosmic rays cutoff at $z \approx 1.6$ [29] [30], and the "cosmological desert" at higher redshifts. In section 5 we propose a novel physical explanation of dark energy, according to which dark energy is simply the energy carried by dark matter. We corroborate our explanation by comparing the predicted amounts of the energy carried by dark matter in different ranges of redshift, with observed results based on $\Lambda \mathrm{CDM}$ cosmologies, and by comparing the predicted equality of matter and dark matter energy densities at redshift $\mathrm{z}=0.5$, with observational data on what is known as the "coincidence problem", namely the observed equality between the densities of matter and dark energy at redshift $z \approx 0.55$ [31] [32] [33]. In Section 6 we summarize and draw main conclusions.

\section{Information Relativity Theory-A Brief Description}

A complete formulation of information relativity theory (IR) and its applications to various field in physics, including small particles physics, quantum mechanics, and cosmology, are detailed elsewhere [26] [27] [28]. For convenience, derivations of the main theory transformations are included in Appendix A.

Before describing the model, and its application to the cosmology of the universe, it is in order here to caution the reader that he or she will probably find difficulty in the absence of the terminology used in the literature. Primarily, we do not use the concept of spacetime. Space and time are treated by us independently, just like Newton did. We refrain from using variables that are not completely understood physically like vacuum energy, or arbitrary parameters like the cosmological constant. All the terms used to construct the theory are observable physical variables.

In principle, information relativity theory is nothing more than "relativizing" Newtonian physics, which we accomplished by accounting for the time travel of information from one reference frame to another. As will become clear from the derivation of the theory's transformations, the scale of the system is of no importance. In several previous articles we showed that not only does the theory 
reproduce quantum theoretical results, including entanglement [34], and particles' wave-like diffraction in the double-slit experiment [35], but it also explains them in simple mechanical terms.

We note that unlike Special Relativity theory, in which the relativity of time is achieved by axiomatizing the constancy of light velocity, relativizing time, and other physical entities in Information Relativity theory is a force majeure of the fact that information does not pass between two points in space instantaneously, but rather suffers delay, which depends on the spatial distance between the two points and the velocity of the information carrier.

The rationale behind the theory is extremely simple and straightforward. It can be illustrated as follows: Consider the case where information from a "moving" body, is transmitted to a "stationary" observer by light signals. Assume that the start and end of an occurrence on the body's reference frame are indicated by two signals sent from the body's "moving" reference frame to the "stationary" observer. Because light's velocity is finite, the two signals will arrive to the observer's reference frame with delays, determined by the distances between the body and the observer at the time when each signal was transmitted. Suppose that the "moving" body is distancing from the observer. In this case, the termination signal will travel a longer distance than the start signal. Thus, the observer will measure a longer occurrence duration than the occurrence duration at the body's reference frame (time dilation). For approaching bodies, the termination signal will travel a shorter distance than the start signal. Thus, the observer will measure a shorter duration than the duration at the body's reference frame (time contraction). Notice that for the above scenario, no synchronization of the clocks at the two reference frames is required.

For the simple case of rectilinear motion with constant velocity $v$, expressing the above mentioned example in the language of mathematics detailed elsewhere [26] [27] [28] (as well as in section 1 in Appendix A) yields the following equation:

$$
\frac{\Delta t}{\Delta t_{0}}=\frac{1}{1-\beta}
$$

where $\Delta t$ is the occurrence's time duration as measured by the observer, $\Delta t_{0}$ is the occurrence's time duration at the body's rest-frame, and $\beta$ is the relative velocity, $\beta=\frac{v}{c}$. Derivations of the transformations of length, mass, and energy, are detailed in Appendix A. The resulting transformations are depicted in the middle column of Table 1 . The transformation in terms of redshift $z$ depicted in the right-hand column will be explained later on. For $\beta \rightarrow 0$ (or $v \ll c$ ), the time, length, matter and energy densities, depicted respectively in the first four rows, reduce to the comparable Newtonian terms. The two new non-classical terms emerging in the model are the dark matter density and its kinetic energy depicted in the last two rows. 
Table 1. Information relativity transformations for inertial rectilinear motion $\left(e_{0}=\frac{1}{2} \rho_{0} c^{2}\right)$.

\begin{tabular}{|c|c|c|c|}
\hline Variable & $\begin{array}{l}\text { Transformation in } \\
\text { terms of velocity }\end{array}$ & $\begin{array}{r}\text { Transformation } i \\
\text { terms of redshif }\end{array}$ & \\
\hline Time interval $\frac{\Delta t}{\Delta t_{0}}$ & $\frac{1}{1-\beta}$ & $z+1$ & $\left(1^{\prime}\right)$ \\
\hline Length $\frac{l}{l_{0}}$ & $\frac{1+\beta}{1-\beta}$ & $2 z+1$ & $\left(2^{\prime}\right)$ \\
\hline Matter density $\frac{\rho_{M}}{\rho_{0}}$ & $\frac{1-\beta}{1+\beta}$ & $\frac{1}{2 z+1}$ & $\left(3^{\prime}\right)$ \\
\hline Matter energy density $\frac{e_{M}}{e_{0}}$ & $\frac{1-\beta}{1+\beta} \beta^{2}$ & $\frac{z^{2}}{(z+1)^{2}(2 z+1)}$ & $\left(4^{\prime}\right)$ \\
\hline Dark matter density $\frac{\rho_{D M}}{\rho_{0}}$ & $\frac{2 \beta}{1+\beta}$ & $\frac{2 z}{2 z+1}$ & $\left(5^{\prime}\right)$ \\
\hline Dark matter energy density $\frac{e_{D M}}{e_{0}}$ & $\frac{2 \beta^{3}}{1+\beta}$ & $\frac{2 z^{3}}{(z+1)^{2}(2 z+1)}$ & $\left(6^{\prime}\right)$ \\
\hline
\end{tabular}

The dark (unobservable) matter density term is defined in the present framework as the difference between the Newtonian and relativistic matter density terms, or:

$$
\frac{\rho_{D M}}{\rho_{0}}=1-\frac{\rho_{M}}{\rho_{0}}=1-\frac{1-\beta}{1+\beta}=\frac{2 \beta}{1+\beta}
$$

Similarly, the energy density carried by the dark matter is defined as the difference between the Newtonian and relativistic matter energy densities:

$$
\frac{e_{D M}}{e_{0}}=\beta^{2}-\frac{1-\beta}{1+\beta} \beta^{2}=\frac{2 \beta^{3}}{1+\beta}
$$

The picture emerging from our analysis thus far suggests that at any given recession velocity, matter and dark matter co-exist in a dynamic duality, with matter decreasing in density and dark matter increasing in density as functions of the recession velocity $\beta$. Thus, in our model matter and dark matter are interchangeable, accelerating the velocity of a receding body of mass is predicted to increase its dark matter at the expense of matter, vice versa, decelerating a receding body of mass is predicted to increase the portion of its matter at the expense of dark matter.

It is worth noting that as $\beta \rightarrow 0$, the density of dark matter and its associated energy $\left(\rho_{D M}, e_{D M}\right)$ approach zero, and Newtonian mechanics under constant rectilinear velocity is recovered. Also, since no restrictions were put on the size of the moving mass, the derived results apply equally well to mesoscopic and microscopic rectilinearly receding bodies of mass. Generalization of the results for motion in a gravitational field is detailed in [28]. In recent papers we demonstrated that Information Relativity theory, without adding any free parameter, 
is successful in predicting and explaining several important cosmological and astrophysical phenomena [26] [36] [37] [38] [39], the neutrino velocity reported by OPERA and four other collaborations [40], as well as quantum phenomena, including quantum entanglement, and the wave-like diffraction patterns of single particles in the double-slit experiment [34] [35].

\section{Dark Matter and Its Dynamical Interaction with Matter, as Functions of the Recession Velocity}

To construct a simple cosmology of the universe we assume a grossly simplified model in which galaxies and galaxy structure are infinitesimal, uncharged, and non-rotating masses, receding from us. We take this as an approximation of the universe at high enough redshifts. For any celestial body receding from us with velocity $\beta$, Figure 1 depicts the densities of observable matter, and dark (unobservable) matter as functions of velocity.

As could be seen, the densities of matter and dark matter are mirror images of one another. They coincide when the recession velocity is exactly $\beta=\frac{v}{c}=\frac{1}{3}$, with matter dominating at recession velocities $\beta<\frac{1}{3}$, and dark matter dominating at recession velocities $\beta>\frac{1}{3}$. As examples, for a recession velocity $\beta=$ 0.01 the model predicts a composition of about $98 \%$ matter and $2 \%$ dark matter, whereas as for a high relativistic velocity of $\beta=0.99$ the predicted composition is $0.5 \%$ matter and $99.5 \%$ dark matter. Metaphorically, we can think of matter and dark matter as two strictly competitive Siamese twins, with one increasing its density at the expense of the other. The ratio of dark matter to matter densities depends only on the recession velocity as depicted in the following equation (see also Figure 2):

$$
\frac{\rho_{D M}}{\rho_{M}}=\frac{2 \beta}{1-\beta}
$$

Figure 3 depicts the matter and dark matter energy density distributions, as functions of the recession velocity $\beta$. As could be seen in the figure, for a receding body of mass, dark matter density increases rapidly with $\beta$. For $\beta=1$, all the energy is stored in the body's dark matter, which travels at a velocity equaling the velocity of light. The matter energy density displays an interesting non-monotonic behavior. It increases with $\beta$ up to a maximum at velocity $\beta=\beta_{c r}$ and then decreases to zero at $\beta=1$. The emerging type of non-monotonicity is quite surprising. No less surprising is the value of $\beta$, at which the matter energy density achieves its unique maximum. Differentiating Equation (4) with respect to $\beta$, and equating the result to zero yields:

$$
\beta^{2}+\beta-1=0
$$

which solves for: 


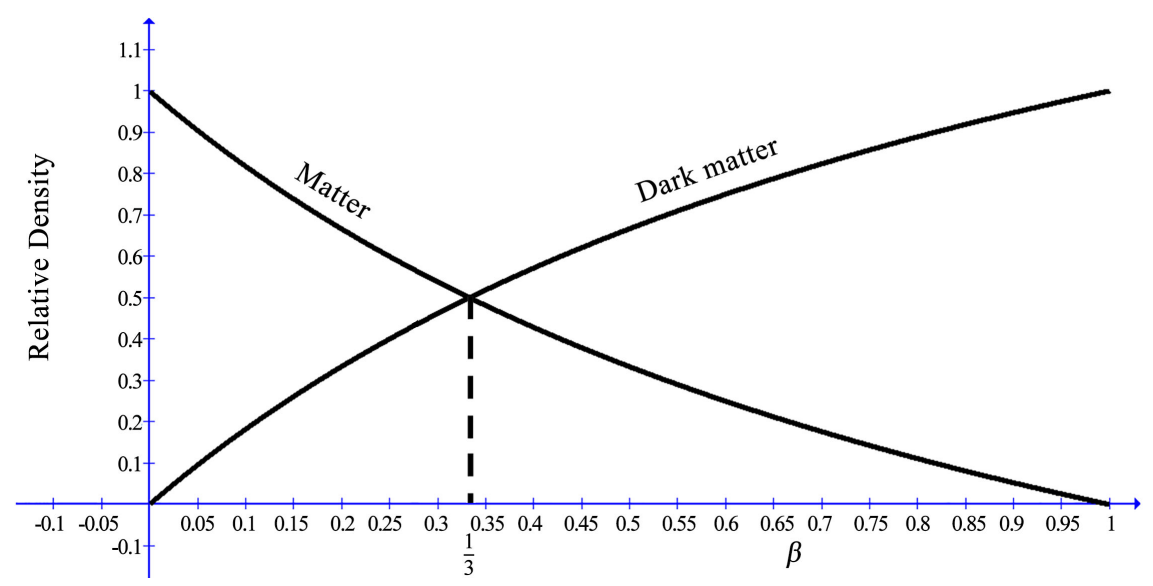

Figure 1. Relative matter and dark matter densities (and their relative energies) as functions of the recession velocity $\beta$.

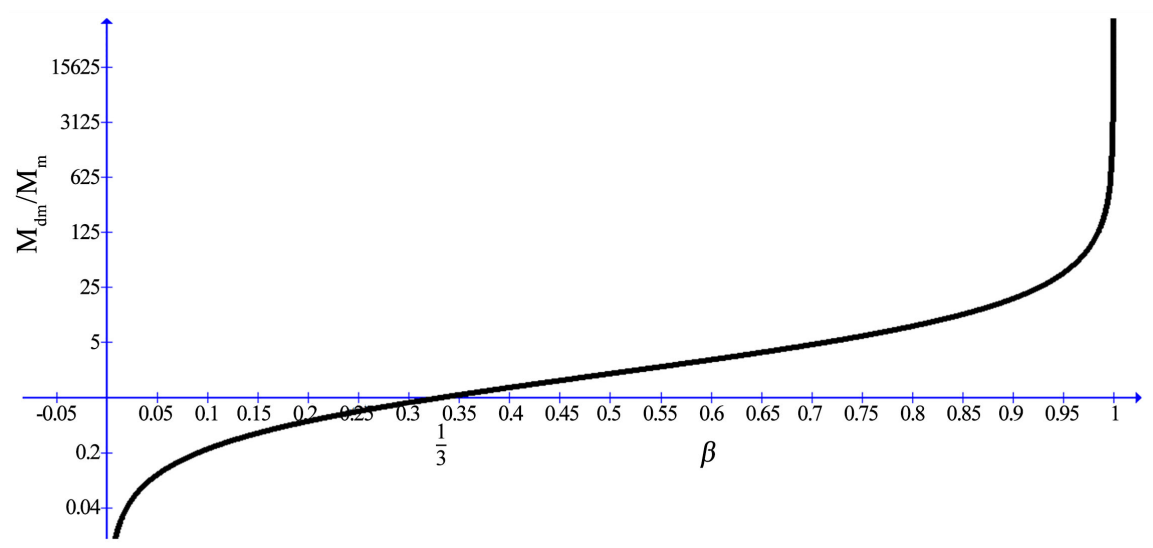

Figure 2. Dark matter to matter ratio as a function of the recession velocity.

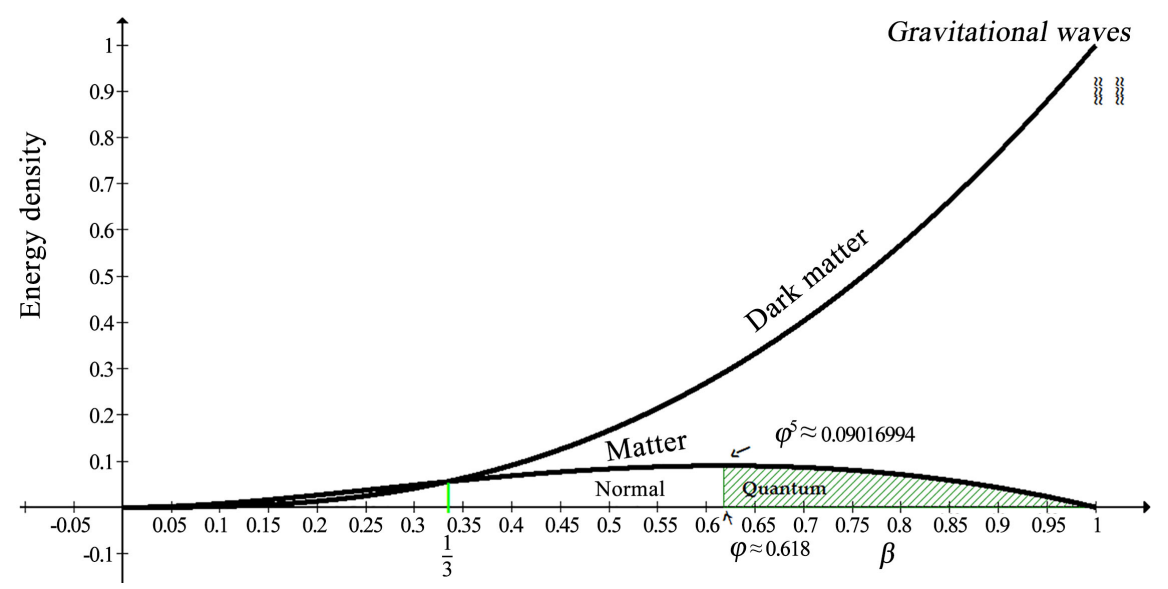

Figure 3. Matter and dark matter energy density distributions, as functions of the recession velocity $\beta$.

$$
\beta_{c r}=\frac{\sqrt{5}-1}{2}=\varphi \approx 0.618
$$

where $\varphi$ is the famous Golden Ratio [41] [42]. Substituting $\beta_{c r}$ in Equation 
(4) yields:

$$
\left(e_{M}\right)_{\max }=e_{0} \varphi^{2} \frac{1-\varphi}{1+\varphi}
$$

From Equation (8) we can write: $\varphi^{2}+\varphi-1=0$, which implies $1-\varphi=\varphi^{2}$ and $1+\varphi=\frac{1}{\varphi}$. Substitution in Equation (10) gives:

$$
\left(e_{M}\right)_{\max }=\varphi^{5} e_{0} \approx 0.09016994 e_{0}
$$

Figure 3 shows clearly that the point $\beta_{c r}=\varphi$ is a point of phase transition, after which matter changes behavior. While for $\beta<\beta_{c r}$ an increase in the recession velocity is associated with increase with the matter's energy, for $\beta>\beta_{c r}$ an increase in velocity is associated with decrease in energy. Quite interestingly, the relative matter energy density at this point, $\frac{\left(e_{M}\right)_{\max }}{e_{0}}$, is equal (to the eighth decimal digit) to Hardy's maximum probability of obtaining an event that contradicts local realism [43]. This equivalence has been underscored by us in a previous publication [26], as well as by Hans Hermann Otto in a recently published paper in this journal [44]. Moreover, the Golden Ratio velocity, at which the phase transition is predicted to occur, echoes nicely with the result of a quantum mechanical experiment published in Science, which demonstrated that applying a magnetic field at right angles to an aligned chain of cobalt niobate atoms, makes the cobalt enter a quantum critical state, in which the ratio between the first two resonances equals the Golden Ratio [45]. Since our theory is scale independent with respect to the moving body's mass, we conjecture, with high certainty, that the critical recession velocity $\beta_{c r}=\varphi \approx 0.618$ is the point of quantum phase transition at cosmological scales.

\section{Dark Matter and Its Dynamical Interaction with Matter as Functions of Redshift}

Since our objective is to apply the theory to the cosmology of the universe, the theory transformations in terms of redshift are added in the right column in Table 1. Their derivation is straightforward, and is detailed elsewhere [26] [28] (see also Appendix B). The relationship between the redshift $z$ and the recession velocity $\beta$ (see Appendix $\mathrm{B}$ ) is found to be:

$$
z=\frac{\beta}{1-\beta}
$$

And the inverse relationship is:

$$
\beta=\frac{z}{z+1}
$$

Substituting Equation (13) in the transformations depicted in the middle column in Table 1 yields the transformation as a function of the redshift $z$ depicted in the right side column of the table. Figure 4 depicts the energy densities 
of matter and dark matter as functions of $z$. The relative densities of matter and dark matter, and their respective energies, are depicted in Figure 5.

As shown in Figure 4, the maximal matter energy density, which marks the point of cosmic quantum phase transition, is predicted to occur at a redshift of $z=1+\varphi \approx 1.618$. This prediction fits quite nicely with the well-known GZK cutoff limit of the cosmic-ray energy spectrum [29] [30], found to exhibit a breakdown in the luminosity densities of both QSO's and AGN's at redshift $z \approx 1.6$. [46] [47], and with a recent discovery of extremely high luminosity galaxies at a redshift of exactly 1.618 [48]. Moreover, as shown in Figure 4 and Figure 5, for the investigated model of the universe, matter is predicted to dominate at redshifts $z<0.5$ (corresponding to recession velocities $\beta<\frac{1}{3}$ ), and dark matter is predicted to dominate at redshifts $z>0.5$.

\section{Dark Energy}

Our model proposes a completely different, and far more plausible explanation about the nature of dark energy. It is not a mysterious negative vacuum energy.

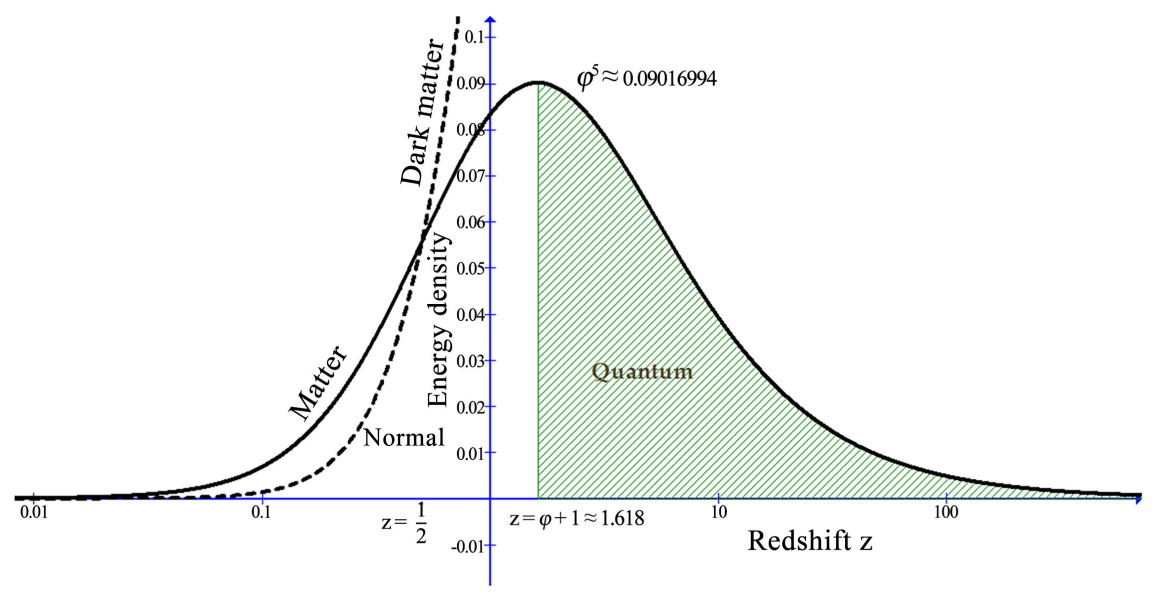

Figure 4. Matter and dark matter energy density distributions as functions of redshift $\mathrm{z}$.

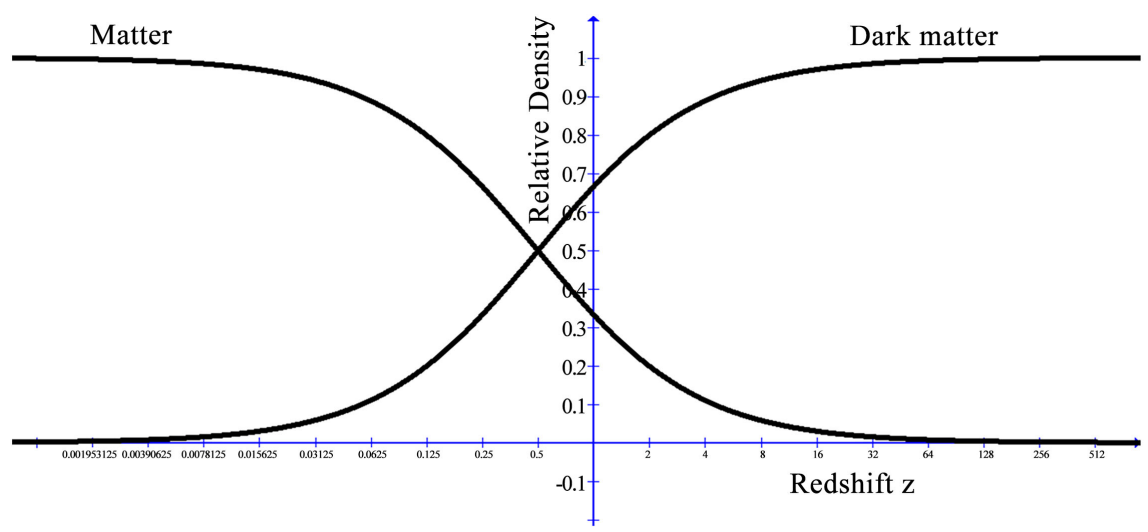

Figure 5. Relative matter and dark matter (and their relative energies) densities as functions of redshift $z$. 
It is the energy carried by the dark matter. Luckily, the name given to dark matter fits well with our explanation. As a post hoc test of our explanation of dark energy, we calculated the proportions of the energies of matter and dark matter at a given redshift range $z$ and compared it with observed results based on $\Lambda \mathrm{CDM}$ cosmologies.

From Equations (4') and (6') in Table 1 we can write:

$$
\frac{e_{M}}{e_{M}+e_{D M}}=\frac{\frac{z^{2}}{(z+1)^{2}(2 z+1)}}{\frac{z^{2}}{(z+1)^{2}(2 z+1)}+\frac{2 z^{3}}{(z+1)^{2}(2 z+1)}}=\frac{1}{1+2 z}
$$

And,

$$
\frac{e_{D M}}{e_{M}+e_{D M}}=\frac{\frac{2 z^{3}}{(z+1)^{2}(2 z+1)}}{\frac{z^{2}}{(z+1)^{2}(2 z+1)}+\frac{2 z^{3}}{(z+1)^{2}(2 z+1)}}=\frac{2 z}{1+2 z}
$$

The ratio of the energy densities of the dark to matter is:

$$
\frac{e_{D M}}{e_{M}}=2 z
$$

The energy of matter and dark matter in any redshift range $\left(z_{1}, z_{2}\right)$ are obtained by integrating expressions 14 and 15 with respect to $Z$, yielding:

$$
\frac{e_{M}\left(z_{1}-z_{2}\right)}{e_{0}}=\frac{1}{2} \ln \left(\frac{2 z_{2}+1}{2 z_{1}+1}\right)-\frac{z_{2}-z_{1}}{\left(z_{2}+1\right)\left(z_{1}+1\right)}
$$

And,

$$
\frac{e_{D M}\left(z_{1}-z_{2}\right)}{e_{0}}=\left(z_{2}-z_{1}\right)+2 \frac{z_{2}-z_{1}}{\left(z_{2}+1\right)\left(z_{1}+1\right)}-2 \ln \left(\frac{z_{2}+1}{z_{1}+1}\right)-\frac{1}{2} \ln \left(\frac{2 z_{2}+1}{2 z_{1}+1}\right)
$$

Calculations based on the above expressions are in good agreement with observations. As an example, it was concluded by Wittman et al. (2000) [49] that for the redshift range 0.6 - 1, dark matter is distributed in a manner consistent with either an open universe, with $\Omega_{b}=0.045, \Omega_{\text {matter }}-\Omega_{b}=0.405, \Omega_{\Lambda}=0$, or with a $\Lambda$ CDM with $\Omega_{b}=0.039, \Omega_{\text {matter }}-\Omega_{b}=0.291, \Omega_{\Lambda}=0.67$, where $\Omega_{b}$ is the fraction of critical density in baryonic matter, $\Omega_{\text {matter }}$ is the fraction of all matter, and $\Omega_{\Lambda}$ is the fraction of dark energy. In the open universe model, we have $\Omega_{\text {matter }}=0.045+0.405=0.45$, and $\Omega_{\Lambda}=0$, whereas in the $\Lambda$ CDM, we have $\Omega_{\text {matter }}=0.039+0.291=0.33$, and $\Omega_{\Lambda}=0.67$. Calculating the ratios of matter and dark matter energies from Equations (17) and (18) for the same redshift range gives:

$$
\frac{e_{M}}{e_{t o t}}=\frac{e_{M}}{e_{M}+e_{D M}}=\frac{0.0300775}{0.0300775+0.0486354} \approx 0.382(\approx 38.2 \%)
$$

And, 


$$
\frac{e_{D M}}{e_{\text {tot }}}=\frac{e_{D M}}{e_{M}+e_{D M}}=\frac{0.0486354}{0.0300775+0.0486354} \approx 0.618(\approx 61.8 \%)
$$

which are in good agreement with the observations based $\Lambda \mathrm{CDM}$ model with $\left(\Omega_{m}=\frac{1}{3}, \Omega_{\Lambda}=\frac{2}{3}\right)$. For the entire range of semi-classical matter $(0 \leq z<1.618)$ we obtain: $\frac{e_{M}}{e_{0}} \approx 0.1038$, and $\frac{e_{D M}}{e_{0}} \approx 0.3420$, yielding:

$$
\frac{e_{M}}{e_{M}+e_{D M}}=\frac{0.138}{0.138+0.3420} \approx 0.233
$$

And,

$$
\left.\frac{e_{D M}}{e_{M}+e_{D M}}=\frac{0.3420}{0.138+0.3420} \approx 0.767 \quad \text { (or } 76.7 \%\right)
$$

Which are in excellent agreement with the $\Lambda$ CDM cosmology with $\Omega_{\text {matter }}=0.23, \Omega_{\Lambda}=0.77$ [50] [51] [52], and quite close to the $\Omega_{\text {matter }}=0.26$, $\Omega_{\Lambda}=0.74$ cosmology [53] [54] [55].

Another supporting evidence for our conjecture that dark energy is the energy carried by dark matter, is the recent observations of what is termed the "coincidence problem", namely, the question why "now" at a redshift $z \approx 0.55$ the densities of matter and dark energy are equal [31] [32] [33]. As Figure 4 and Figure 5 clearly show, the energy densities of matter and dark matter are predicted to be equal at $z=0.5$ (corresponding to a recession velocity of $\beta=\frac{1}{3}$ ). Considering the simplified assumptions made in the discussed model of the universe, the theoretical prediction is in good agreement with the "coincidence problem" observational data.

\section{Summary and Main Conclusions}

The nature and properties of dark matter and dark energy in the universe are among the outstanding open issues of modern cosmology. The tremendous effort to answer the question "what is dark matter made of?" has so far been unsatisfactory. Most effort was, and is still being, invested in searches for particle dark matter candidates, partly within the Standard Model of elementary particles, but mostly among its supersymmetric extensions.

The different approach taken here is based on our recently proposed Information Relativity theory [26] [27] [28]. Rather than theorizing about the elementary particles that might qualify as candidates for dark matter, we investigated the relativistic effects on large-scale cosmological structures in their recession in the universe. We defined the mass of dark matter as the complimentary portion of the relativistic mass, such that at any given recession velocity the sum of the two is equal to the Newtonian mass. The emerging picture from our model could be summarized as follows:

1) For a body in recession, matter and dark matter co-exist in a dynamic dual- 
ity, with matter decreasing in density, and dark matter increasing in density as functions of the recession velocity $\beta$ (and Redshift $z$ ) (see Figure 1 and Figure 5). We conjecture that at recession velocity $\beta \rightarrow 1(v \rightarrow c)$, which corresponds to redshift of $z \rightarrow \infty$, dark matter transforms to a gravitational wave.

2) Contrary to the current conjecture that dark energy is something different than dark matter, a mysterious negative vacuum energy of unknown source, our model suggests that dark energy is the energy carried by dark matter. With respect to the discovery that the universe expands with acceleration [56] [57], we propose that the observed acceleration is not a result of a negative energy of yet unknown source, but rather the aftermath of the decrease (as function of $\frac{1}{r^{2}}$ ) in the gravitation pulls between galaxies as they become more distant from one another. Metaphorically speaking, we contend that the universe is accelerating, not because someone is pressing on the gasoline pedal, but because he or she is gradually releasing the pressure from the brake pedal.

3) At a recession velocity $\beta$ equaling the Golden Ratio $(\approx 0.618)$, or equivalently $z \approx 1.618$, matter undergoes a critical quantum phase transition (see Figure 3 and Figure 4). This prediction provides an explanation, at least partly, to the GZK cosmic rays cutoff at redshifts $z \approx 1$.6. According to our model, up to the critical point, the universe is comprised of matter and dark matter, whereas at redshifts higher than $\approx 1.618$ the "cosmic desert" is comprised of quantum matter and dark matter (see Figure 4). It has been recently argued that the difficulty in tracing the Hubble diagram of the universe in the high redshift range of above $z=1.7$ is a limitation of the use of supernovas as calibration candles, due to their limited intrinsic luminosity and extinction from the interstellar medium. Luminosity correlations of long gamma-ray bursts (GRBs) were proposed as a complementary method for high redshifts [58]. Our analysis suggests that the "cosmological desert" at high redshift is not only a measurement problem, which could be overcome with further refinements of existing measurement methods, but is mainly attributed to the quantum nature of matter at redshifts higher than $\sim 1.618$.

4) Matter dominates the universe at $z<0.5$, and dark matter dominates at $z>$ 0.5 . In good agreement with observational data, the two components are predicted to be equal at $z=0.5$. This could provide a physical explanation to the "coincidence problem", namely the question why "now", at a redshift $z \approx 0.55$, the densities of matter and dark energy are equal [31] [32] [33].

5) The emergence of the Golden Ratio symmetry in our analysis as a key player in the structure of the Universe, echoes nicely with many discovered Golden Ratio symmetries in nature, science, technology, and the arts, including in the structure of plants [59] [60] [61], quantum matter [45] [62] [63], the human brain [64], human economic behavior [65] [66] [67] [68], music [69] [70], and aesthetics [71].

We note that the emerging duality between matter and dark matter holds 
much in common with the realistic matter-wave duality in quantum mechanics, first conjectured by Louis de Broglie [72]. In the de Broglie-Bohm model of quantum mechanics [73] [74] [75] [76], the dual wave of a fast traveling or spinning particle is real, piloting its dual corpuscular mass. Similarly, in our theory dark matter is unobservable matter accompanying its dual observable matter. For extremely high velocities $(\beta \rightarrow 1)$, matter diminishes, while dark matter, which carries most of the total energy, becomes wave-like, just like de Broglie's pilot wave. Moreover, our model suggests that the recently detected gravitational waves [77] [78], believed to be disturbance in the "fabric of spacetime", are fluctuations in real wave-like dark matter, traveling at quasi-luminal velocities. The possibility that LIGO had in fact detected dark matter has been recently suggested [79].

It is worthwhile to stress that the cosmology proposed here is based on a very simplified model, in which galaxies, or even galaxy-clusters, are represented as point masses receding rectilinearly along the line of sight of an observer on Earth. Real galaxies and galaxy structures are in rotation relative to an Earth's observer, and the stars and planets in each galaxy are also in continuous rotation. The observed rotations of all celestial structures are major sources of dark and quantum matter. The process of their production due to the structures' rotational motion could be analyzed in a similar way to the current analysis. We have already embarked on the analysis of the production of dark and quantum matter due to rotational motion, and of the spatial entanglement between the dark matter dispersed spherically at their halos. We conjecture that the physical entanglement of dark matter in space is medium by which gravitational forces between bodies are enacted. It is well accepted that dark matter is the "gravitational glue" that keeps galaxies and galaxy structures bound together. Our preliminary analysis confirms this.

\section{Acknowledgements}

I wish to thank Dr. Raid Saabni and three anonymous reviewers for their helpful remarks.

\section{Conflicts of Interest}

The authors declare no conflicts of interest regarding the publication of this paper.

\section{References}

[1] Barack, L., et al. (2018) White Paper for the COST Action "Gravitational Waves, Black Holes, and Fundamental Physics. 269 p. arXiv:1806.05195

[2] Bertone, G. and Silk, J. (2010) Particle Dark Matter. In: Bertone, G., Ed., Particle Dark Matter. Observations, Models and Searches, Cambridge University Press, Cambridge.

[3] Turner, M.S. (1993) Dark Matter: Theoretical Perspectives. PNAS, 90, 4827-4834. 
https://doi.org/10.1073/pnas.90.11.4827

[4] Bertone, G., Hooper, D. and Silk, J. (2005) Particle Dark Matter: Evidence, Candidates and Constraints. Physics Reports, 405, 279-390.

https://doi.org/10.1016/j.physrep.2004.08.031

[5] Clowe, D., et al. (2006) A Direct Empirical Proof of the Existence of Dark Matter. The Astrophysical Journal, 648, L109-L113. https://doi.org/10.1086/508162

[6] Clowe, D., Randall, S.W. and Markevitch, M. (2007) Catching a Bullet: Direct Evidence for the Existence of Dark Matter. Nuclear Physics B-Proceedings Supplements, 173, 28-31. https://doi.org/10.1016/j.nuclphysbps.2007.08.150

[7] Bergström, L. (2000) Non-Baryonic Dark Matter: Observational Evidence and Detection Methods. Reports on Progress in Physics, 63, 793. https://doi.org/10.1088/0034-4885/63/5/2r3

[8] Savage, C., et al. (2009) Compatibility of DAMA/LIBRA Dark Matter Detection with Other Searches. Journal of Cosmology and Astroparticle Physics. https://doi.org/10.1088/1475-7516/2009/04/010.

[9] Feng, L.L. (2010) Dark Matter Candidates from Particle Physics and Methods of Detection. Annual Review of Astronomy and Astrophysics, 48, 495-545. https://doi.org/10.1146/annurev-astro-082708-101659

[10] Ellis, J. (2003) Dark Matter and Dark Energy: Summary and Future Directions. Philosophical Transactions of the Royal Society A, 361, 2607-2627.

[11] Steigman, G. and Turner, M.S. (1985) Cosmological Constraints on the Properties of Weakly Interacting Massive Particles. Nuclear Physics B, 253, 375-386. https://doi.org/10.1016/0550-3213(85)90537-1

[12] Sivertsson, S. and Gondolo, P. (2011) The WIMP Capture Process for Dark Stars in the Early Universe. The Astrophysical Journal, 729, 51.

[13] Aprile, E., et al. (2011) Dark Matter Results from 100 Live Days of XENON100 Data. Physical Review Letters, 107, 131302.

[14] Griest, K. (2006) WIMPs and MACHOs. Encyclopedia of Astronomy \& Astrophysics. P. Murdin \& IOP Publishing Ltd.

[15] Alcock, C. (1998) EROS and MACHO Combined Limits on Planetary-Mass Dark Matter in the Galactic Halo. The Astrophysical Journal, 499, 9-12. https://doi.org/10.1086/311355

[16] Alcock, C. (2001) MACHO Project Limits on Black Hole Dark Matter in the 1-30 Solar Mass Range. The Astrophysical Journal Letters, 550, 169-172. https://doi.org/10.1086/319636

[17] Carr, B., Kuhnel, F. and Sandstad, M. (2016) Primordial Black Holes as Dark Matter. Physical Review D, 94, Article ID: 083504. https://doi.org/10.1103/PhysRevD.94.083504

[18] Albrecht, A., et al. (2006) Report of the Dark Energy Task Force.

[19] Linder, E.V. (2004) Probing Gravitation, Dark Energy, and Acceleration. Physical Review D, 70, Article ID: 023511. https://doi.org/10.1103/PhysRevD.70.023511

[20] Alam, U., Sahni, V. and Starobinsky, A.A. (2004) The Case for Dynamical Dark Energy Revisited. Journal of Cosmology and Astroparticle Physics, 2004, 008. https://doi.org/10.1088/1475-7516/2004/06/008

[21] Sandvik, H.B., Barrow, J.D. and Magueijo, J. (2002) A Simple Cosmology with a Varying Fine Structure Constant. Physical Review Letters, 88, Article ID: 031302. https://doi.org/10.1103/PhysRevLett.88.031302 
[22] Easson, D.A. Framptona, P.H. and Smoota, G.F. (2011) Entropic Accelerating Universe. Physics Letters B, 696, 273-277. https://doi.org/10.1016/j.physletb.2010.12.025

[23] Wang, B., Abdalla, E., Atrio-Barandela, F. and Pavón, D. (2016) Dark Matter and Dark Energy Interactions: Theoretical Challenges, Cosmological Implications and Observational Signatures. Reports on Progress in Physics, 79, Article ID: 096901. https://doi.org/10.1088/0034-4885/79/9/096901

[24] Cai, R.-G. (2005) Cosmology with Interaction between Phantom Dark Energy and Dark Matter and the Coincidence Problem. Journal of Cosmology and Astroparticle Physics, 2005, 002. https://doi.org/10.1088/1475-7516/2005/03/002

[25] Farrar, G.R. and Peebles, P.J.E. (2004) Interacting Dark Matter and Dark Energy. The Astrophysical Journal, 604, 1-11. https://doi.org/10.1086/381728

[26] Suleiman, R. (2013) The Dark Side Revealed: A Complete Relativity Theory Predicts the Content of the Universe. Progress in Physics, 4, 34-40.

[27] Suleiman, R. (2016) Information Relativity Theory Solves the Twin Paradox Symmetrically. Physics Essays, 29, 304-308. https://doi.org/10.4006/0836-1398-29.3.304

[28] Suleiman, R. (2017) Information Relativity: The Special and General Theory. https://www.researchgate.net/publication/312214434

[29] Greisen, K. (1966) End to the Cosmic-Ray Spectrum? Physical Review Letters, 16, 748-750. https://doi.org/10.1103/PhysRevLett.16.748

[30] Zatsepin, G.T. and Kuz'min, V.A. (1966) Upper Limit to the Spectrum of Cosmic Rays. Journal of Experimental and Theoretical Physics Letters, 4, 78-80.

[31] Zlatev, I., Wang, L. and Steinhardt, P.J. (1999) Quintessence, Cosmic Coincidence, and the Cosmological Constant. Physical Review Letters, 82, 896. https://doi.org/10.1103/PhysRevLett.82.896

[32] Poitra, V. (2014) Can the Coincidence Problem Be Solved by a Cosmological Model of Coupled Dark Energy and Dark Matter? General Relativity and Gravitation, 46, 1732. https://doi.org/10.1007/s10714-014-1732-0

[33] Velten, H.E.S., vom Marttens, R.F. and Zimdahl, W. (2014) Aspects of the Cosmological "Coincidence Problem". The European Physical Journal, 74, 3160. https://doi.org/10.1140/epjc/s10052-014-3160-4

[34] Suleiman, R. (2015) If God Plays Dice, Must We Do the Same? Quantum Entanglement as a Deterministic Phenomenon. Poster Presented at the Fourth International Conference on New Frontiers in Physics (ICNFP 2015), Kolymbari, Crete, 24-30 August 2015. http://vixra.org/pdf/1505.0147v4.pdf

[35] Suleiman, R. (2016) A Relativistic Model of Matter-Wave Duality Explains the Results of the Double-Slit Experiment. 5 th International Conference on New Frontiers in Physics, Kolymbari, 6-14 July 2016, 1-14. https://www.researchgate.net/publication/310844720

[36] Suleiman, R. (2017) Outside Spacetime III: An Axiom-Free Relativizing of Newtonian Physics Predicts the Black Holes' Schwarzschild Radius (without Interior Singularities). Unpublished Manuscript.

[37] Suleiman, R. (2017) Outside Spacetime: An Axiom-Free Relativizing of Newtonian Physics Predicts the Degree of Solar Light Bending, as Good as General Relativity. Unpublished Manuscript.

[38] Suleiman, R. (2017) An Axiom-Free Relativizing of Newtonian Physics Predicts the Gravitational Redshift as Good as General Relativity. Unpublished Manuscript. 
[39] Suleiman, R. (2018) A Non-Axiomatic Modification of Newton's Second Law Predicts the Anomalous Increase in Acceleration at Large Distances and High Velocities. Unpublished Manuscript.

[40] Suleiman, R. (2014) A Relativistic Newtonian Mechanics Predicts with Precision the Results of Recent Neutrino-Velocity Experiments. Journal of Advances in Physics, 6, 1032-1035.

[41] Olsen, S. (2006) The Golden Section. Walker and Co., New York.

[42] Livio, M. (2002) The Golden Ratio: The Story of Phi, the World's Most Astonishing Number. Broadway Books, New York.

[43] Hardy, L. (1994) Nonlocality of a Single Photon Revisited. Physical Review Letters, 73, 2279-2283. https://doi.org/10.1103/PhysRevLett.73.2279

[44] Otto, H.H. (2018) Reciprocity Relation between the Mass Constituents of the Universe and Hardy's Quantum Entanglement Probability. World Journal of Condensed Matter Physics, 8, 30-35. https://doi.org/10.4236/wjcmp.2018.82003

[45] Coldea, R., Tennant, D.A., Wheeler, E.M., Wawrzynska, E., Prabhakaran, D., Telling, M., Habicht, K., Smeibidl, P. and Kiefer, K. (2010) Quantum Criticality in an Ising Chain: Experimental Evidence for Emergent E8 Symmetry. Science, 327, 177-180. https://doi.org/10.1126/science.1180085

[46] Bergman, D.R., et al. (2006) Can Experiments Studying Ultrahigh Energy Cosmic Rays Measure the Evolution of the Sources?

[47] Thomson, G. (2006) The TA and TALE Experiments. Journal of Physics: Conference Series, 47, 248-254. https://doi.org/10.1088/1742-6596/47/1/030

[48] Gilli, R., et al. (2003) Tracing the Large-Scale Structure in the Chandra Deep Field South. The Astrophysical Journal, 592, 721-727. https://doi.org/10.1086/375777

[49] Wittman, D.M., et al. (2000) Detection of Weak Gravitational Lensing Distortions of Distant Galaxies by Cosmic Dark Matter at Large Scales. Nature, 405, 143-148. https://doi.org/10.1038/35012001

[50] Samushia, L. and Ratra, B. (2009) Constraints on Dark Energy Models from Radial Baryon Acoustic Scale Measurements. The Astrophysical Journal, 701, 1373-1380. https://doi.org/10.1088/0004-637X/701/2/1373

[51] Farooq, M.O. (2013) Observational Constraints on Dark Energy Cosmological Model Parameters.

[52] Kunz, M. and Bassett, B.A. (2004) A Tale of Two Distances.

[53] Furlanetto, S.R. (2006) The Global 21-Centimeter Background from High Redshifts. Monthly Notices of the Royal Astronomical Society, 371, 867-878. https://doi.org/10.1111/j.1365-2966.2006.10725.x

[54] Oguri, M., et al. (2008) The Sloan Digital Sky Survey Quasar Lines Search. III Constrains on Dark Energy from the Third Data Released Quasar Lens Catalog. The Astronomical Journal, 135, 512-519. https://doi.org/10.1088/0004-6256/135/2/512

[55] Viel, M., Haehnelt, M.G. and Springel, V. (2004) Inferring the Dark Matter Power Spectrum from the Lyman $\alpha$ Forest in High-Resolution QSO Absorption Spectra. Monthly Notices of the Royal Astronomical Society, 354, 684-694. https://doi.org/10.1111/j.1365-2966.2004.08224.x

[56] Riess, A.G., et al. (1998) Observational Evidence from Supernovae for an Accelerating Universe and a Cosmological Constant. The Astronomical Journal, 116, 1009-1038. https://doi.org/10.1086/300499

[57] Perlmutter, S., et al. (1999) Measurements of $\Lambda$ and $\Omega$ from 42 High-Redshift Su- 
pernovae. The Astronomical Journal, 517, 565-586. https://doi.org/10.1086/307221

[58] Wang, G.-J., Yu, H., Li, Z.-X., Xia, J.-Q. and Zhu, Z.-H. (2017) Evolutions and Calibrations of Long Gamma-Ray-Burst Luminosity Correlations Revisited. The Astrophysical Journal, 103, 103-111. https://doi.org/10.3847/1538-4357/aa5b9b

[59] Douady, S. and Couder, Y. (1992) Phyllotaxis as a Physical Self-Organizing Growth Process. Physical Review Letters, 68, 2098-2101. https://doi.org/10.1103/PhysRevLett.68.2098

[60] Klar, A.J.S. (2002) Fibonacci's Flowers. Nature, 417, 595. https://doi.org/10.1038/417595a

[61] Zeng, L. and Wang, G. (2009) Modeling Golden Section in Plants. Progress in Natural Science, 19, 255-260. https://doi.org/10.1016/j.pnsc.2008.07.004

[62] Stoudenmire, E.M., Clarke, D.J., Mong, R.S.K. and Alicea, J. (2015) Assembling Fibonacci Anyons from a Z3 Parafermion Lattice Model. Physical Review B, 91, Article ID: 235112.

[63] Lindner, J.F., Kohar, V., Kia, B., Hippke, M., Learned, J.G. and Ditto, W.L. (2015) Strange Nonchaotic Stars. Physical Review Letters, 114, Article ID: 054101. https://doi.org/10.1103/PhysRevLett.114.054101

[64] Kopell, N.J. and Whittington, M.A. (2008) Temporal Interactions between Cortical Rhythms. Frontiers in Neuroscience, 2, 145-154. https://doi.org/10.3389/neuro.01.034.2008

[65] van den Bos, K., Cropanzano, R., Kirk, J., Jasso, G. and Okimoto, T.G. (2015) Expanding the Horizons of Social Justice Research: Three Essays on Justice Theory. Social Justice Research, 28, 229-246. https://doi.org/10.1007/s11211-015-0237-7

[66] Suleiman, R. (2017) Economic Harmony: An Epistemic Theory of Economic Interactions. Games, 8, 2. https://doi.org/10.3390/g8010002

[67] Schuster, S. (2017) A New Solution Concept for the Ultimatum Game Leading to the Golden Ratio. Scientific Reports, 7, Article No. 5642. https://doi.org/10.1038/s41598-017-05122-5

[68] Suleiman, R. (2018) On Gamesmen and Fairmen: Explaining Fairness in Noncooperative Bargaining Games. Royal Society Open Science, 5, Article ID: 171709. https://doi.org/10.1098/rsos.171709

[69] Hammel, G.T. and Vaughan, K.C. (1995) Math and Music: Harmonious Connections. Dale Seymour Publications.

[70] Putz, J.F. (1995) The Golden Section and the Piano Sonatas of Mozart. Mathematics Magazine, 68, 275-282. https://doi.org/10.1080/0025570X.1995.11996333

[71] Pittard, N., Ewing, M. and Jevons, C. (2007) Aesthetic Theory and Logo Design: Examining Consumer Response to Proportion across Cultures. International Marketing Review, 24, 457-473. https://doi.org/10.1108/02651330710761026

[72] De Broglie, L. (1925) Researches on the Quantum Theory.

[73] De Broglie, L. (1923) Waves and Quanta. Nature, 112, 540. https://doi.org/10.1038/112540a0

[74] De Broglie, L. (1970) The Reinterpretation of Wave Mechanics, Foundations of Physics, 1, 5-15. https://doi.org/10.1007/BF00708650

[75] Bohm, D. (1952) A Suggested Interpretation of the Quantum Theory in Terms of "Hidden" Variables, I. Physical Review, 85, 166-179. https://doi.org/10.1103/PhysRev.85.166

[76] Bohm, D. (1952) A Suggested Interpretation of the Quantum Theory in Terms of 
“Hidden” Variables, II. Physical Review, 85, 180-193.

https://doi.org/10.1103/PhysRev.85.180

[77] Abbott, B.P., et al. (LIGO Scientific Collaboration and Virgo Collaboration) (2016) Observation of Gravitational Waves from a Binary Black Hole Merger. Physical Review Letters, 116, Article ID: 061102.

https://doi.org/10.1103/PhysRevLett.116.061102

[78] Abbott, B.P., et al. (LIGO Scientific Collaboration and Virgo Collaboration) (2017) GW170817: Observation of Gravitational Waves from a Binary Neutron Star Inspiral. Physical Review Letters, 119, Article ID: 161101. https://doi.org/10.1103/PhysRevLett.119.161101

[79] Bird, S., et al. (2016) Did LIGO Detect Dark Matter? Physical Review Letters, 116, Article ID: 201301. https://doi.org/10.1103/PhysRevLett.116.201301 


\section{Appendix A}

\section{Derivation of Information Theory's Transformations}

In sections 1a-4a we derive the theories transformations of time, length, matter density, and energy density, as functions of the recession velocity $\beta$.

\section{1a. Time Transformation}

Consider two reference frames, $F$ and $F^{\prime}$, moving with constant velocity $v$ with respect to each other. A "stationary" observer in frame $F$ defines events with coordinates $t, x, y, z$. Another observer in $F^{\prime}$ defines events using the coordinates $t^{\prime}, x^{\prime}, y^{\prime}, z^{\prime}$. For simplicity, assume that the coordinate axes in each frame are parallel ( $x$ is parallel to $x^{\prime}, y$ to $y^{\prime}$, and $z$ to $z^{\prime}$ ), and that the two systems are synchronized, such that at $t=t^{\prime}=0,(x, y, z)=\left(x^{\prime}, y^{\prime}, z^{\prime}\right)=(0,0,0)$. Now consider the case in which a physical occurrence starts at the point of origin in $F^{\prime}$ at $t_{1}^{\prime}=0$, and lasts for a period of $\Delta t^{\prime}=t_{2}^{\prime}-t_{1}^{\prime}=t^{\prime}-0=t^{\prime}$, as measured by an observer at the occurrence rest frame in $F^{\prime}$. We use the term occurrence to denote a physical time-dependent process. By this we seek to differentiate it from the term "event", which in physics came to mean an occurrence that is sharply localized at a single point in space and instant of time. The observer at $F$ has no way of knowing when the occurrence at $F^{\prime}$ ended, unless information is sent to him/her from the observer at $F^{\prime}$ indicating the termination of the occurrence. Such information could be sent by any type of information carrier as long as its velocity, $V_{\mathcal{c}}$ exceeds the relative velocity $v$ at which $F^{\prime}$ is departing from $F$, i.e., $V_{c}$ should satisfy $V_{c}>v$. After $t$ seconds for an observer in $F$, the reference frame $F^{\prime}$ will be at distance $x=v t$. Thus, the information about the termination of the physical occurrence will arrive to the observer at $F$ with a delay of:

$$
t_{d}=\frac{x}{V_{c}}=\frac{v t}{V_{c}} .
$$

The termination time registered by the observer at $F$ will be

$$
t=t^{\prime}+\frac{v t}{V_{c}}
$$

which could be written as:

$$
t=\frac{1}{1-\frac{v}{V_{c}}} t^{\prime}=\frac{1}{1-\beta} t^{\prime},
$$

where $\beta=\frac{v}{V_{c}}$.

Quite interestingly, Equation (3a), is quite similar to the Doppler's Formula derived for the frequency modulation of waves emitted from traveling bodies. Importantly, in both cases the direction of motion matters. In the Doppler Effect a wave emitted from a distancing body will be red-shifted (longer wavelength), whereas a wave emitted from an approaching body with be blues-shifted (shorter wavelength). The degree of red, or blue shift is an increasing function of the 
body's velocity. The same applies to the time duration of an occurrence taking place at a stationary point on a moving frame. If the frame is distancing from $F$, the time measured at $F$ will be dilated relative to the time measured at frame $F^{\prime}$, whereas if the frame is approaching $F$, the time measured at $F$ will contract.

It is important to note further that the above derived transformation applies to all carriers of information, including the commonly employed acoustic and optical communication media. For the case in which information is carried by light, with velocity $\mathcal{c}$, or by electromagnetic waves with equal velocity, Equation (3a) becomes:

$$
\frac{\Delta t}{\Delta t^{\prime}}=\frac{1}{1-\frac{v}{c}}=\frac{1}{1-\beta}
$$

where $\beta=\frac{v}{c}$.

Evidently, the derived result does not agree with the time dilation prescribed by Special Relativity under the assumption of constancy of the velocity of light in vacuum. We prefer here to avoid the above mentioned inconsistency, by strictly limiting our theorizing to cases in which the information carrier wave propagates in velocities $c<c_{0}$, where $c_{0}$ is the velocity of light in vacuum. Putting such a limit does depreciate the value of our result, since it will still apply to all physical situations of interest.

\section{2a. Length Transformation}

To derive the distance transformation, assume that $F^{\prime}$ has on board a rod placed along its $x^{\prime}$ axis between the points $x^{\prime}=0$ and $x^{\prime}=x_{2}^{\prime}$, and that the observer in $F^{\prime}$ uses his clock to measure the length of the rod (in its rest frame) and communicates his measurement to the observer in $F$. Assume that the information carrier from frame $F^{\prime}$ to frame $F$ travels with constant velocity $V_{c}$ (as measured in the source rest frame). To perform the measurement of the rod's length, at $t_{1}^{\prime}=t_{1}=0$, a signal is sent from the rare end of the rod, i.e., from $x^{\prime}=x_{2}^{\prime}$ to the observer at the point of origin $x^{\prime}=0$. The length in $F^{\prime}$ (the rest frame) is calculated as:

$$
l_{0}=x_{2}^{\prime}=V_{c} t_{2}^{\prime} .
$$

Denote by $\Delta t_{c}$ is the time duration in the signal's rest frame for its arrival to the observer in $F^{\prime}$. Using Equation (3a), $t_{2}^{\prime}$ as a function of $\Delta t_{c}$ can be expressed as:

$$
t_{2}^{\prime}=\frac{1}{1-\frac{-v}{V_{c}}} \Delta t_{c}=\frac{1}{1+\frac{v}{V_{c}}} \Delta t_{c},
$$

which could be rewritten as:

$$
\Delta t_{c}=\left(1+\frac{v}{V_{c}}\right) t_{2}^{\prime} .
$$


Because $F^{\prime}$ is departing $F$ with velocity $V$, the signal will reach an observer in $F$ at time $t_{2}$ equaling

$$
t_{2}=\Delta t_{c}+\frac{v t_{2}}{V_{c}}=\Delta t_{c}+\frac{v}{V_{c}} t_{2}
$$

Substituting the value of $\Delta t_{c}$ from Equation (7a) in Equation (8a) yields:

$$
t_{2}=\left(1+\frac{v}{V_{c}}\right) t_{2}^{\prime}+\frac{v}{V_{c}} t_{2}
$$

which could be rewritten as:

$$
t_{2}=\frac{\left(1+\frac{v}{V_{c}}\right)}{\left(1-\frac{v}{V_{c}}\right)} t_{2}^{\prime} .
$$

Substituting the value of $t_{2}^{\prime}$ from Equation (5a), we get:

$$
t_{2}=\frac{\left(1+\frac{v}{V_{c}}\right)}{\left(1-\frac{v}{V_{c}}\right)} \frac{l_{0}}{V_{c}} .
$$

Thus, the observer in $F$ will conclude that the length of the rod is equal to

$$
l=V_{c} t_{2}=\frac{\left(1+\frac{v}{V_{c}}\right)}{\left(1-\frac{v}{V_{c}}\right)} l_{0}
$$

or,

$$
\frac{l}{l_{0}}=\frac{1+\beta}{1-\beta}
$$

where $\beta=\frac{v}{V_{c}}$.

Regardless of the value of $V_{c}$, the above derived relativistic distance equation predicts distance contraction only when the two reference frames approach each other (i.e., for $-1<\beta \leq 0$ ). On the other hand, in contradiction of the famous Lorentz contraction, for distancing frames (i.e., $0<\beta<1$ ) Equation (13a) predicts length extension.

\section{3a. Mass Transformation}

Let us assume that that the rod has a total rest-mass $m_{0}$ distributed uniformly along the $x$ axis. According to Equation 13a an approaching rod will contract causing the mass density along the $x$ axis to increase. On the other hand, a distancing rod will extend, causing its mass density along the $x$ axis to dilute. The body's mass density in its rest-frame is $\rho_{0}=\frac{m_{0}}{A l_{0}}$, where $A$ is the area of the 
body's cross section, perpendicular to the direction of movement. In $F$ the density is given by: $\rho_{M}=\frac{m_{0}}{A l}$, where $l$ is the object's length in $F$. Using the length transformation (Equation (13a)) we can write:

$$
\rho_{M}=\frac{m_{0}}{A l}=\frac{m_{0}}{A l_{0}\left(\frac{1+\beta}{1-\beta}\right)}=\rho_{0} \frac{1-\beta}{1+\beta}
$$

Or,

$$
\frac{\rho_{M}}{\rho_{0}}=\frac{1}{l / l_{0}}=\frac{1-\beta}{1+\beta}
$$

For the case in which information is carried by waves traveling at the velocity of light we set $\beta=\frac{v}{c}$. As could be seen from Equation (15a), the relativistic mass density is inversely proportional to the relativistic length. It is predicted to increase for approaching bodies and a decrease for distancing bodies. For applications to cosmology, the unobservable (Dark) matter is defined as the difference between the Newtonian, and relativistic mass densities, or:

$$
\frac{\rho_{D M}}{\rho_{0}}=1-\frac{1-\beta}{1+\beta}=\frac{2 \beta}{1+\beta}
$$

Figure 1 in the main text depicts the densities of observable and unobservable (dark) matter as functions of velocity. As could be seen in the figure, the theory predicts that the densities of observable and unobservable matter become equal at velocity of recession equaling exactly $\beta=\frac{v}{c}=\frac{1}{3}$. The ratio of dark matter to matter densities, is given by:

$$
\frac{\rho_{D M}}{\rho_{M}}=\frac{2 \beta}{1-\beta}
$$

\section{4a. Energy Transformation}

The matter energy density of a moving body with velocity $v$, and rest density $\rho_{0}$ is given by:

$$
e_{M}=\frac{1}{2} \rho v^{2}=\frac{1}{2} \rho_{0} c^{2} \frac{1-\beta}{1+\beta} \beta^{2}=e_{0} \frac{1-\beta}{1+\beta} \beta^{2}
$$

where $e_{0}=\frac{1}{2} \rho_{0} c^{2}$. For $\beta \rightarrow 0$ (or $v \ll c$ ) Equation (18a) reduces to $e=\frac{1}{2} \rho_{0} v^{2}$, which is the classical Newtonian expression.

Figure 1a depicts the relativistic matter energy density as a function of velocity $\beta$. The dashed line depicts the corresponding Newtonian relationship. For approaching bodies, the theory predicts an increase with $\beta$, up to infinity as $\beta \rightarrow-1$. Strikingly, for distancing bodies, the matter energy density displays a non-monotonic behavior. It increases with $\beta$ up to a maximum at velocity $\beta=\beta_{c r}$, and then decreases to zero at $\beta=1$. The emerging type of 
non-monotonicity is quite surprising. No less surprising is the value of $\beta$, at which the matter energy density achieves its unique maximum. Differentiating Equation 18a with respect to $\beta$, and equating the result to zero yields:

$$
\beta^{2}+\beta-1=0
$$

which solves for:

$$
\beta_{c r}=\frac{\sqrt{5}-1}{2}=\varphi \approx 0.618
$$

where $\varphi$ is the famous Golden Ratio [1a] [2a]. Substituting $\beta_{c r}$ in Equation (18a) yields:

$$
\left(e_{M}\right)_{\max }=e_{0} \varphi^{2} \frac{1-\varphi}{1+\varphi}
$$

From Equation (19a) we can write: $\varphi^{2}+\varphi-1=0$, which implies $1-\varphi=\varphi^{2}$ and $1+\varphi=\frac{1}{\varphi}$. Substitution in Equation (21a) gives:

$$
\left(e_{M}\right)_{\max }=\varphi^{5} e_{0} \approx 0.09016994 e_{0}
$$

The results in Equations (20a) and (22a) are quite amazing, given the key role played by the Golden Ratio in nature, in nature, technology and the arts, including in the structure of plants, physics, the structure of the human brain, music, aesthetics, the social sciences, and more (see relevant references in the main text).

The energy carried by dark matter is given by:

$$
e_{D M}=\frac{1}{2} \rho_{D M} v_{c}^{2}=\frac{1}{2} \rho_{D M} c^{2} \beta^{2}=\left(\frac{1}{2} \rho_{0} c^{2}\right) \frac{2 \beta}{1+\beta} \beta^{2}=\left(\frac{1}{2} \rho_{0} c^{2}\right) \frac{2 \beta^{3}}{1+\beta}
$$

Or:

$$
\frac{e_{D M}}{e_{0}}=\frac{2 \beta^{3}}{1+\beta}
$$

where $e_{0}=\frac{1}{2} \rho_{0} c^{2}$.

The predicted dark matter energy density increases rapidly with velocity (see Figure 3 in the main text). At relatively low velocities, the bulk of the body's energy is carried by its observable matter, while at high relativistic velocities, the bulk of the body's energy is carried by its dark matter. The energy carried by matter, and the energy carried by dark matter are predicted to be equal precisely at $\beta=\frac{1}{3}$. The model predicts that at $\beta_{c r}=\varphi=0.618$ a receding body undertakes a phase transition, seizing to behave classically. The value of the matter energy density at this point reaches a peak equal to $\varphi^{5} e_{0}$, which amounts to $\approx$ $0.09016994 e_{0}$. The dark matter energy density at this critical point becomes equal to $\frac{2 \varphi^{3}}{1+\varphi}=2 \varphi^{4} \approx 0.29179607$. These are aesthetically beautiful results, which could be put to direct experimental test. 


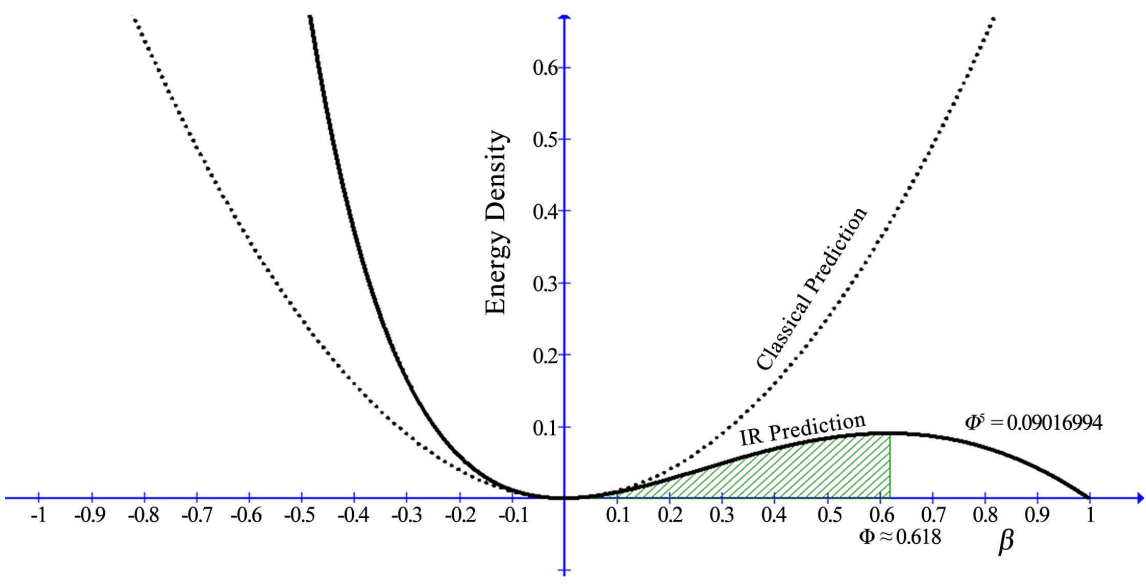

Figure 1a. Matter energy density as a function of the recession velocity.

\section{References}

[1a] Olsen, S. (2006) The Golden Section. Walker \& Co., New York.

[2a] Livio, M. (2002) The Golden Ratio: The Story of Phi, the World's Most Astonishing Number. Broadway Books, New York. 


\section{Appendix B}

\section{Relationship between Recession Velocity and Redshift}

To derive the expression of redshift in its dependence on the recession velocity, consider an observer on earth who receives redshifted waves emitted from a receding celestial object (e.g., A star, galaxy center, etc.). Assume that the recession velocity of the celestial object at the time the light wave was emitted was equal to $v$. Using Doppler's formula, we can write:

$$
z=\frac{\lambda_{o b}-\lambda_{e m}}{\lambda_{e m}}=\frac{f_{e m}-f_{o b}}{f_{o b}}
$$

where $\lambda_{e m}\left(f_{e m}\right)$ is the wavelength (frequency) of the wave emitted by the object and $\lambda_{o b}\left(f_{o b}\right)$ is the wavelength (frequency) measured by the observer. We also have $f_{e m}=\frac{1}{\Delta t_{e m}}$ and $f_{o b}=\frac{1}{\Delta t_{o b}}$, where $\Delta t_{e m}$ and $\Delta t_{o b}$ are the time intervals corresponding to $f_{e m}$ and $f_{o b}$, respectively. Substitution in Equation (1b) gives:

$$
z=\frac{\frac{1}{\Delta t_{e m}}-\frac{1}{\Delta t_{o b}}}{\frac{1}{\Delta t_{o b}}}=\frac{\Delta t_{o b}}{\Delta t_{e m}}-1
$$

From Equation (1) in the main text we have: $\frac{\Delta t_{o b}}{\Delta t_{e m}}=\frac{1}{1-\beta}$, where $\beta=\frac{v}{c}$. Substitution in Equation ( $2 \mathrm{~b}$ ) yields:

$$
z=\frac{1}{1-\beta}-1=\frac{\beta}{1-\beta}
$$

And the recession velocity in terms of redshift is:

$$
\beta=\frac{z}{z+1}
$$

For blue-shift the same equation holds except that we must replace $\beta$ by $-\beta$. 